\title{
Clinical Implications of Claudin18.2 Expression in Patients With Gastric Cancer
}

\author{
JIN HO BAEK ${ }^{1}$, DONG JIN PARK ${ }^{2}$, GYU YEOL KIM ${ }^{2}$, JAEKYUNG CHEON ${ }^{3}$, \\ BYUNG WOOG KANG ${ }^{1}$, HEE JEONG CHA ${ }^{4}$ and JONG GWANG KIM ${ }^{1}$ \\ ${ }^{1}$ Department of Oncology/Hematology, Kyungpook National University Chilgok Hospital, \\ School of Medicine, Kyungpook National University, Daegu, Republic of Korea; \\ ${ }^{2}$ Department of Surgery, Ulsan University Hospital, \\ University of Ulsan College of Medicine, Ulsan, Republic of Korea; \\ ${ }^{3}$ Department of Hematology and Oncology, Ulsan University Hospital, \\ University of Ulsan College of Medicine, Ulsan, Republic of Korea; \\ ${ }^{4}$ Department of Pathology, Ulsan University Hospital, \\ University of Ulsan College of Medicine, Ulsan, Republic of Korea
}

\begin{abstract}
Background/Aim: Claudin18.2 (CLDN18.2) is a tight junction protein that has been identified as a promising target in gastric cancer. This study aimed to evaluate the clinical relevance of CLDN18.2 expression in gastric cancer. Patients and Methods: This study included 367 patients diagnosed with gastric cancer, who underwent curative surgical resection. Immunohistochemical staining for CLDN18.2 was carried out, and expression was scored semi-quantitatively, based on staining intensity and the percentage of staining. Results: CLDN18.2 expression was observed in 273 patients (74.4\%), and 108 (29.4\%) were classified as CLDN18.2-positive by predefined criteria. CLDN18.2 expression was not correlated with age, sex, tumor location, or stage. Expression rates were higher in diffusetype and HER2-positive tumors. In multivariate survival analysis, CLDN18.2 expression was not associated with survival outcomes. Conclusion: Higher expression of CLDN18.2 was observed in diffuse-type and HER2-positive gastric cancers. Meanwhile, CLDN18.2 expression was not associated with survival in patients with gastric cancer.
\end{abstract}

Correspondence to: Hee Jeong Cha, MD, Ph.D., Department of Pathology, Ulsan University Hospital, University of Ulsan College of Medicine, 877 Bangeojinsunhwandoro, Dong-gu, Ulsan 44033, Republic of Korea. Tel: +82 522528241, Fax: +82 522507048, e-mail: heej0124@uuh.ulsan.kr; Jong Gwang Kim, MD, Ph.D., Department of Oncology/Hematology, Kyungpook National University Chilgok Hospital, School of Medicine, Kyungpook National University, 807 Hogukno, Buk-gu, Daegu 41404, Republic of Korea. Tel: +82 532002623, Fax: +82 532002029, e-mail: jkk21c@knu.ac.kr

Key Words: Claudin18.2 (CLDN18.2), clinical implications, expression rate, gastric cancer.
Gastric cancer is the fifth most common malignancy and the third-leading cause of cancer-related mortality worldwide, even though rapid advances in treatment options have improved its prognosis (1). Recently, remarkable progress in tumor biology has led to the development of new therapeutics that target critical aspects of oncogenic pathways or the immune system. In advanced gastric cancer (AGC), various targeted agents have already been evaluated in randomized studies, where trastuzumab [anti-human epidermal growth factor receptor 2 (HER2) monoclonal antibody] exhibited anti-tumor activity against $15-20 \%$ of HER2-positive AGCs (2), while ramucirumab [anti-vascular endothelial growth factor receptor 2 (VEGFR2) monoclonal antibody] and nivolumab [anti-programmed cell death protein 1 (PD-1) monoclonal antibody] improved survival duration in a second- or third-line setting $(3,4)$. However, most responses to chemotherapy with targeted agents are limited and of short duration: median survival is 10-16 months, and overall survival (OS) at 2 years rarely exceeds $10 \%$ (5). Given these results, the development of new targeted agents with improved efficacy and reasonable toxicity, and which extend the treatment possibilities in AGC, is urgently needed.

The claudin (CLDN) family of transmembrane proteins has a crucial role in the formation of tight junctions and comprises at least 27 members (6). CLDNs are associated with multimolecular complexes and transduction of cell signaling pathways (7), and have also been reportedly associated with regulation of proliferation and differentiation through interactions with signaling proteins (8). Previous studies have reported that the expression levels of CLDNs are altered in various cancers compared to normal tissues (9). Among CLDNs, claudin18.2 (CLDN18.2) is not expressed 
in any healthy tissues, except the stomach mucosa, but is broadly expressed in gastric cancers, especially of the diffuse type (10).

Zolbetuximab (formerly known as IMAB362) is a monoclonal antibody specific to CLDN18.2. A recent randomized phase II study demonstrated that the addition of zolbetuximab prolonged survival and improved objective response rate relative to chemotherapy alone. Therefore, CLDN18.2 was identified as a promising treatment target in patients with AGC or gastro-esophageal junction cancer and CLDN18.2 overexpression (11).

In previous studies, the expression rate of CLDN18.2 was found to be inconsistent. In a Japanese study, the positive rate of CLDN18.2 was observed in $52 \%$ of primary gastric tumors and $45 \%$ of lymph node metastases (12). However, positive rates of $42.2 \%$ and $48.0 \%$ were reported in Caucasian studies $(11,13)$. Moreover, CLDN18.2 expression was not associated with survival outcomes (13).

Accordingly, the present study investigated the clinical relevance and prognostic impact of CLDN18.2 expression in a large population of Korean patients with localized gastric cancer who underwent surgical resection.

\section{Patients and Methods}

Patients and treatment. This study retrospectively reviewed 938 patients diagnosed with gastric cancer and who underwent curative surgical resection at Ulsan University Hospital between January 2012 and December 2017. The patients were enrolled according to the following criteria: 1) pathologically diagnosed with primary gastric adenocarcinoma; and stage II or III gastric cancer classified by the American Joint Committee on Cancer staging (7th edition) (14). A total of 268 patients met these criteria and were included in the study. Another 99 patients with stage I gastric cancer were included for comparing the expression status of CLDN18.2 by stage. Patient records were also reviewed for data regarding medical history, age, sex, adjuvant chemotherapy, surgical methods, and pathologic results.

The adjuvant chemotherapy for stage II or III patients started 46 weeks after surgery with tegafur/gimeracil/oteracil (15) or capecitabine/oxaliplatin (16). Observation without adjuvant therapy was also an option in the elderly population or patients with Eastern Cooperative Oncology Group (ECOG) performance status $\geq 3$.

Immunohistochemical staining of CLDN18.2 and CLDN11. An immunohistochemical (IHC) study for CLDN18.2 (Abcam, Cambridge, UK; 1:75) was carried out on formalin-fixed paraffin, with a $4-\mu \mathrm{m}$ thick serial section of tissue embedded using the BOND-MAX system (Leica Biosystems, Wetzlar, Germany), with a bond polymer refine red detection kit, according to the manufacturer's recommended protocol.

The expression of CLDN18.2 was scored semi-quantitatively, based on staining intensity and the percentage of staining. Staining intensity was subclassified as: 0 , negative; 1 , weak; 2 , moderate; and 3 , strong. The proportion of staining was scored as: 0 , negative; $1,1-10 \% ; 2,11-50 \%$; and $3,51-100 \%$. Cases with a percentage of staining score of 3 (51-100\%), and with moderate to strong staining intensity ( 2 or 3 ), were defined as positive. The pathologist, without prior clinical or pathologic information, scored expression at $100 \times$ magnification under light microscopy. All available areas in the section were evaluated. In the original study design, we also planned to evaluate CLND11 expression, but this expression was very weak in a preliminary study. Therefore, we did not evaluate CLND11 expression.

Statistical analysis. Descriptive statistics are reported as incidences and percentages. Associations between categoric variables were evaluated using the Chi-squared test. Disease-free survival (DFS) was calculated from the date of surgery to the date of tumor recurrence or death from any cause. OS was measured from the date of surgery to death from any cause. In event-free subjects, data were censored at the last follow-up. Survival curves were calculated by the Kaplan-Meier method and were compared by the log-rank test. Multivariate analysis of prognostic factors was carried out using Cox's proportional hazard regression model. The hazard ratio (HR) and $95 \%$ confidence interval (CI) were estimated for each factor. A $p$-value $<0.05$ was considered statistically significant. The statistical analyses were performed using SPSS for Windows (version 20.0; SPSS Inc., Chicago, IL, USA).

\section{Results}

Patient and tumor characteristics. Patient and tumor characteristics are summarized in Table I. The median age was 60 years (range $=30-93$ years) at the time of surgery, and 253 patients $(69.8 \%)$ were male. Primary tumors were located in the upper stomach in 52 patients $(14.2 \%)$, middle stomach in 124 (33.8\%), and lower stomach in $191(52.0 \%)$. According to tumor staging, 123 cases $(33.5 \%)$ were stage III, 145 (39.5\%) were stage II, and 99 (27.0\%) were stage I. According to Lauren phenotype, 265 patients (72.2\%) had intestinal-type, and $96(26.2 \%)$ had diffuse-type. Two hundred and seventy-two patients $(74.1 \%)$ were HER2 0 or $1+$, and 95 (25.9\%) were HER2 $2+$ or $3+$, by IHC. Additional fluorescence in situ hybridization (FISH) results were not available. We defined HER2 $2+$ or $3+$ cases as positive. Forty-two $(29.0 \%)$ and 67 patients $(46.2 \%)$ in stage II, and $63(51.2 \%)$ and $31(25.2 \%)$ in stage III, received capecitabine/oxaliplatin and tegafur/gimeracil/oteracil as adjuvant chemotherapy, respectively.

CLDN18.2 expression status. Overall, CLDN18.2 expression was observed in 273 patients (74.4\%), and 108 (29.4\%) were classified as positive by predefined criteria. In 74 cases (20.2\%), weak (1) staining intensity was observed; and 153 $(41.7 \%)$ and 46 cases $(12.5 \%)$ were moderate (2) and strong (3), respectively. The cases of proportion of staining were as follows: $1,1-10 \%$ in 49 cases $(13.4 \%) ; 2,11-50 \%$ in 102 cases $(27.8 \%)$; and $3,51-100 \%$ in 122 cases $(33.2 \%)$.

Association between CLDN18.2 expression and clinicopathologic features. CLDN18.2 staining intensity, staining proportion, and expression status were not correlated with age, sex, and primary tumor location. $\mathrm{T}$ stage, $\mathrm{N}$ stage, and tumor, 
Table I. Patient characteristics and CLDN18.2 expression.

\begin{tabular}{|c|c|c|c|c|c|c|c|c|c|c|c|c|c|c|}
\hline \multirow[t]{2}{*}{ Variables } & \multirow{2}{*}{$\begin{array}{c}\text { Total } \\
(n=367)\end{array}$} & \multicolumn{5}{|c|}{ Staining intensity } & \multicolumn{5}{|c|}{ Staining proportion } & \multicolumn{3}{|c|}{ CLND18.2 expression } \\
\hline & & 0 & 1 & 2 & 3 & $p$-Value & 0 & 1 & 2 & 3 & $p$-Value & $\begin{array}{c}\text { Negative } \\
(\mathrm{n}=259 \\
70.6 \%)\end{array}$ & $\begin{array}{l}\text { Positive } \\
(\mathrm{n}=108, \\
29.4 \%)\end{array}$ & $p$-Value \\
\hline \multicolumn{15}{|l|}{ Age } \\
\hline Median (range) & $60(30-93)$ & & & & & & & & & & & $61(30-93)$ & $60(32-87)$ & 0.388 \\
\hline Gender & & & & & & 0.728 & & & & & 0.306 & & & 0.719 \\
\hline Male & 253 & 70 & 50 & 95 & 38 & & 70 & 31 & 71 & 81 & & 180 & 73 & \\
\hline Female & 114 & 24 & 24 & 58 & 8 & & 24 & 18 & 31 & 41 & & 79 & 35 & \\
\hline \multicolumn{2}{|c|}{ Primary tumor location } & & & & & 0.097 & & & & & 0.314 & & & 0.303 \\
\hline Upper & 52 & 11 & 7 & 20 & 14 & & 11 & 6 & 14 & 21 & & 32 & 20 & \\
\hline Middle & 124 & 34 & 28 & 49 & 13 & & 34 & 22 & 27 & 41 & & 89 & 35 & \\
\hline Lower & 191 & 49 & 39 & 84 & 19 & & 49 & 21 & 61 & 60 & & 138 & 53 & \\
\hline $\mathrm{T}$ stage & & & & & & 0.488 & & & & & 0.033 & & & 0.192 \\
\hline $\mathrm{T} 1$ & 107 & 16 & 34 & 48 & 9 & & 16 & 17 & 33 & 41 & & 71 & 36 & \\
\hline $\mathrm{T} 2$ & 45 & 13 & 6 & 20 & 6 & & 13 & 4 & 11 & 17 & & 29 & 16 & \\
\hline $\mathrm{T} 3$ & 107 & 35 & 15 & 38 & 19 & & 35 & 13 & 31 & 28 & & 81 & 26 & \\
\hline $\mathrm{T} 4$ & 108 & 30 & 19 & 47 & 12 & & 30 & 15 & 27 & 36 & & 78 & 30 & \\
\hline $\mathrm{N}$ stage & & & & & & 0.700 & & & & & 0.108 & & & 0.497 \\
\hline No & 156 & 33 & 42 & 68 & 13 & & 33 & 24 & 44 & 55 & & 108 & 48 & \\
\hline N1 & 80 & 19 & 12 & 36 & 13 & & 19 & 11 & 23 & 27 & & 55 & 25 & \\
\hline $\mathrm{N} 2$ & 66 & 22 & 9 & 23 & 12 & & 22 & 3 & 21 & 20 & & 49 & 17 & \\
\hline N3 & 65 & 20 & 11 & 26 & 8 & & 20 & 11 & 14 & 20 & & 47 & 18 & \\
\hline TNM stage & & & & & & 0.318 & & & & & 0.008 & & & 0.181 \\
\hline I & 99 & 17 & 29 & 48 & 5 & & 17 & 15 & 28 & 39 & & 65 & 34 & \\
\hline II & 145 & 34 & 29 & 56 & 26 & & 34 & 19 & 46 & 46 & & 103 & 42 & \\
\hline III & 123 & 43 & 16 & 49 & 15 & & 43 & 15 & 28 & 37 & & 91 & 32 & \\
\hline Lauren phenotype & & & & & & 0.063 & & & & & $<0.001$ & & & 0.001 \\
\hline Intestinal & 265 & 83 & 41 & 105 & 36 & & 83 & 34 & 76 & 72 & & 199 & 66 & \\
\hline Diffuse & 96 & 10 & 33 & 44 & 9 & & 10 & 15 & 26 & 45 & & 59 & 37 & \\
\hline Unclassified & 6 & 1 & 0 & 4 & 1 & & 1 & 0 & 0 & 5 & & 1 & 5 & \\
\hline HER2 status & & & & & & $<0.001$ & & & & & 0.003 & & & 0.009 \\
\hline 0 or $1+$ & 272 & 79 & 60 & 108 & 25 & & 79 & 38 & 74 & 81 & & 202 & 70 & \\
\hline $2+$ or $3+$ & 95 & 15 & 14 & 45 & 21 & & 15 & 11 & 28 & 41 & & 57 & 38 & \\
\hline
\end{tabular}

CLND18.2: Claudin18.2; TNM stage: tumor, node, metastasis stage; HER2: human epidermal growth factor receptor 2.

node, metastasis (TNM) stage were not correlated with CLDN18.2 staining intensity or expression status, but staining percentages showed a decreasing relationship with advanced $\mathrm{T}$ stage and TNM stage. CLDN18.2 expression by stage was as follows: $34.3 \%$ in stage I, $29.0 \%$ in stage II, and $26.0 \%$ in stage III. A slightly decreasing tendency was detected, but there was no statistically significant correlation between CLDN18.2 expression and TNM stage. Lauren phenotype and HER2 status were correlated with CLDN18.2 expression status. Lauren diffuse-type showed an increasing association with staining percentage and higher positive expression rate. CLDN18.2 staining intensities and percentage showed increasing trends in HER2-positive (2+ or 3+) cases, and the CLDN18.2 expression rate was significantly higher in HER2-positive than HER2negative ( 0 or $1+)$ patients.
CLDN18.2 expression and survival outcomes. With a median follow-up of 917 (115-2571) days, estimated 5-year DFS and OS rates were $73.7 \%$ and $74.7 \%$, respectively. Patients aged $\geq 60$ years had significantly lower OS $(p=0.044)$, and statistically insignificantly lower DFS $(p=0.127)$, than patients aged $<60$ years. Female patients had significantly lower DFS ( $p=0.018)$, and statistically insignificantly lower OS $(p=0.080)$, than male patients. There were statistically significant differences in DFS $(p<0.001)$ and OS $(p<0.001)$ according to TMN stage. However, there were no statistically significant differences in DFS and OS according to primary tumor location, Lauren phenotype, or HER2 status. Also, there were no statistically significant differences in DFS $(p=0.878)$ and OS $(p=0.914)$ according to CLDN18.2 expression status (Table II). In the multivariate analysis, 
Table II. Kaplan-Meier cumulative disease-free survival and overall survival.

\begin{tabular}{|c|c|c|c|c|c|}
\hline Variables & $\begin{array}{l}\text { No. of } \\
\text { patients }\end{array}$ & $\begin{array}{l}5-\mathrm{yrs} \\
\text { DFS } \\
(\%)\end{array}$ & $p$-Value & $\begin{array}{c}5-\mathrm{yrs} \\
\text { OS } \\
(\%)\end{array}$ & $p$-Value \\
\hline Overall & 367 & 73.7 & & 74.7 & \\
\hline Age & & & 0.127 & & 0.044 \\
\hline$<60$ & 172 & 79.1 & & 82.1 & \\
\hline$\geq 60$ & 195 & 68.6 & & 68.1 & \\
\hline Gender & & & 0.018 & & 0.080 \\
\hline Male & 253 & 77.9 & & 78.1 & \\
\hline Female & 114 & 64.2 & & 67.9 & \\
\hline Primary tumor location & & & 0.174 & & 0.103 \\
\hline Upper & 52 & 68.6 & & 63.5 & \\
\hline Middle & 124 & 74.7 & & 76.4 & \\
\hline Lower & 191 & 74.7 & & 76.7 & \\
\hline TNM stage & & & $<0.001$ & & $<0.001$ \\
\hline I & 99 & & & & \\
\hline (3-yrs DFS) & & 97.0 & & & \\
\hline (3-yrs OS) & & & & 98.0 & \\
\hline II & 145 & 85.7 & & 87.0 & \\
\hline III & 123 & 47.1 & & 52.5 & \\
\hline Lauren phenotype & & & 0.639 & & 0.471 \\
\hline Intestinal & 265 & 73.6 & & 76.2 & \\
\hline Diffuse & 96 & 71.0 & & 70.6 & \\
\hline HER2 status & & & 0.062 & & 0.088 \\
\hline 0 or $1+$ & 272 & 71.0 & & 72.4 & \\
\hline $2+$ or $3+$ & 95 & 84.3 & & 83.2 & \\
\hline CLDN18.2 expression & & & 0.878 & & 0.914 \\
\hline Negative & 259 & 75.1 & & 75.6 & \\
\hline Positive & 108 & 68.0 & & 69.6 & \\
\hline
\end{tabular}

DFS: Disease-free survival; OS: overall survival; TNM stage: tumor, node, metastasis stage; HER2: human epidermal growth factor receptor 2; CLND18.2: claudin18.2.

female sex and TNM were identified as independent prognostic factors for DFS $(p=0.002$ and $p<0.001$, respectively) and OS ( $p=0.022$ and $p<0.001$, respectively). In multivariate survival analysis, CLDN18.2 expression status was not an independent prognostic factor for DFS or OS (Table III).

\section{Discussion}

In the current study, we investigated the clinical significance of CLDN18.2 expression in localized gastric cancer patients who underwent gastrectomy. Our results showed that CLDN18.2 expression rates were higher in diffuse-type $(p=0.001)$ and HER2-positive $(2+/ 3+; p=0.007)$ cancers, but CLDN18.2 expression was not correlated with TNM stage. Meanwhile, there were no statistically significant differences in survival outcomes according to CLDN18.2 expression status.
The positive expression rate of CLDN18.2 was $29.4 \%$ in the present study, which was lower than that in previous studies. In the randomized, phase II, FAST study, $48 \%$ of patients were considered to have a positive expression (11). The CLAUDETECT $^{\text {TM }} 18.2$ kit (Ganymed Pharmaceuticals, Mainz, Germany) was used for IHC, and moderate to strong CLDN18.2 expression and membrane staining intensity $\geq 2+$ in $\geq 40 \%$ of cancer cells were defined as positive expression. Rohde et al. (12) reported that $52 \%$ of primary gastric tumors, and $45 \%$ of lymph node metastases, showed moderate to strong CLDN18.2 expression in Japanese patients with the CLAUDETECT ${ }^{\mathrm{TM}} 18.2$ kit. Dottermusch et al. (13) also reported CLDN18.2 expression in $42.2 \%$ of gastric cancer cases in a Caucasian cohort using an anti-CLDN18.2 antibody from Abcam; these researchers used histoscore for expression scoring. In the current study, we used a semi-quantitative scoring method for staining intensity and percentage of staining. We defined cases with a percentage of staining score of $3(51-100 \%)$ and with moderate to strong staining intensity ( 2 or 3 ) as positive. Moreover, an Abcam monoclonal antibody against CLDN18.2 was used due to unavailability of the CLAUDETECT ${ }^{\mathrm{TM}} 18.2$ kit. Given these results, some differences in expression rates may be due to differences in monoclonal antibodies used for detection, stages, and predefined criteria. Plus, ethnic differences need to be further evaluated.

In our study, the expression rate of CLDN18.2 was higher in diffuse-type $(p=0.001)$, but not correlated with age, sex, primary tumor location, or TNM stage. Associations between CLDN18.2 expression and clinicopathologic features were reported in some studies. Rohde et al. (12) reported that CLDN18.2 expression was significantly higher in diffuse-type and high-grade (G3) tumors than in other tumors. These investigators also reported that the fraction of tumor cells expressing CLDN18.2 at any staining intensity correlated strongly with the primary tumor and lymph node metastases, but $\mathrm{T}$ stage and $\mathrm{N}$ stage were not associated with CLDN18.2 expression. CLDN18-ARHGAP26 fusions have been identified in gastric cancers, with a predominance in diffuse-type gastric cancers $(17,18)$. Tanaka et al. (19) reported that almost all CLDN18-ARHGAP26 fusion-positive cancers showed positive CLDN18 immunostaining and CLDN18 translocation, and are significantly characterized by CLND18 overexpression.

The expression rate of CLDN18.2 was also higher in HER2 positive $(2+/ 3+; p=0.007)$ tumors in the current study. However, Dottermusch et al. (13) reported that CLDN18.2 expression was not correlated with HER2 status or MET status. Sahin et al. (10) demonstrated that activation of human CLDN18.2 depends on binding of the transcription factor cAMP-responsive element-binding protein (CREB) to its unmethylated consensus site. An important role for HER2/neu has been suggested for CREB expression and activity. Steven et al. (20) also reported a positive correlation of HER-2/neu and pCREB in breast cancer lesions by IHC 
Table III. Multivariate analysis of disease-free survival and overall survival.

\begin{tabular}{|c|c|c|c|c|c|c|}
\hline & \multicolumn{3}{|c|}{ DFS } & \multicolumn{3}{|c|}{ OS } \\
\hline & HR & $95 \% \mathrm{CI}$ & $p$-Value & HR & $95 \% \mathrm{CI}$ & $p$-Value \\
\hline Age $(<60 v s . \geq 60)$ & 1.204 & $0.754-1.924$ & 0.436 & 1.534 & $0.890-2.645$ & 0.124 \\
\hline Gender (Male vs. Female) & 2.115 & $1.328-3.367$ & 0.002 & 1.863 & $1.094-3.172$ & 0.022 \\
\hline TNM stage & & & $<0.001$ & & & $<0.001$ \\
\hline I vs. II & 4.787 & $1.090-21.024$ & 0.038 & 5.236 & $0.669-40.949$ & 0.115 \\
\hline I vs. III & 30.288 & $7.365-124.567$ & $<0.001$ & 32.721 & $4.479-239.026$ & 0.001 \\
\hline $\begin{array}{l}\text { CLDN18.2 expression } \\
\text { (Negative vs. Positive) }\end{array}$ & 1.221 & $0.733-2.033$ & 0.443 & 1.146 & $0.621-2.113$ & 0.663 \\
\hline
\end{tabular}

DFS: Disease-free survival; OS: overall survival; HR: hazard ratio; CI: confidence interval; TNM stage: tumor, node, metastasis stage; CLND18.2: claudin18.2.

staining. Accordingly, further studies are warranted to validate the associations between CLDN18.2 and HER2 in gastric cancer. The co-expression of CLDN18.2 and HER2 was observed in $25.9 \%$ of patients in the present study and in $13.8 \%$ in the FAST study (21). Moran et al. (22) have also reported that $12 \%$ of cases showed CLDN18.2 and HER2 co-expression. Accordingly, dual targeting strategy (antiHER2 and anti-CLDN18.2 monoclonal antibodies) could be a possible treatment option for these patients with coexpression of CLDN18.2 and HER2.

Some reports suggest that CLDN18.2 expression decreases as cancer progresses, contributing to the invasive potential of tumor cells and to metastasis $(23,24)$. Although, there were no correlations between CLDN18.2 expression and TNM stage or survival in our study, Jun et al. reported that reduced CLDN18 expression correlated with perineural invasion and poor overall survival (23). Therefore, further studies are needed to clarify the role of CLDN18.2 in gastric cancer progression and survival.

In summary, CLDN18.2 expression was positive in $29.4 \%$ of patients by predefined criteria in the present population. Although, CLDN18.2 expression was not associated with survival outcomes, CLDN18.2 expression rates were higher in diffuse-type and HER2 positive (2+/3+). Accordingly, further studies are warranted to standardize detection methods and to validate the clinical significance of CLDN18.2 in gastric cancer.

\section{Conflicts of Interest}

The Authors have no conflicts of interest to declare regarding this study.

\section{Authors' Contributions}

JH Baek contributed to data acquisition, analysis, drafting and revising the manuscript. DJ Park and GY Kim performed surgical treatments and contributed to critically revising and approving the final version of the manuscript. $J$ Cheon contributed to data acquisition and critically revising and approving the final version of the manuscript. BW Kang contributed to data analysis and critically revising and approving the final version of the manuscript. HJ Cha examined pathological findings and contributed to analysis, drafting and revising the manuscript. JG Kim conceived and designed the study, interpreted the data, and contributed to drafting, critically revising and approving the final version of the manuscript.

\section{Acknowledgements}

This work was supported by the National Research Foundation of Korea (NRF) grant funded by the Korea government (2014R1A5A 2009242).

\section{References}

1 Fitzmaurice C, Allen C, Barber RM, Barregard L, Bhutta ZA, Brenner H, Dicker DJ, Chimed-Orchir O, Dandona R, Dandona L, Fleming T, Forouzanfar MH, Hancock J, Hay RJ, HunterMerrill R, Huynh C, Hosgood HD, Johnson CO, Jonas JB, Khubchandani J, Kumar GA, Kutz M, Lan Q, Larson HJ, Liang X, Lim SS, Lopez AD, MacIntyre MF, Marczak L, Marquez N, Mokdad AH, Pinho C, Pourmalek F, Salomon JA, Sanabria JR, Sandar L, Sartorius B, Schwartz SM, Shackelford KA, Shibuya K, Stanaway J, Steiner C, Sun J, Takahashi K, Vollset SE, Vos T, Wagner JA, Wang H, Westerman R, Zeeb H, Zoeckler L, AbdAllah F, Ahmed MB, Alabed S, Alam NK, Aldhahri SF, Alem G, Alemayohu MA, Ali R, Al-Raddadi R, Amare A, Amoako Y, Artaman A, Asayesh H, Atnafu N, Awasthi A, Saleem HB, Barac A, Bedi N, Bensenor I, Berhane A, Bernabe E, Betsu B, Binagwaho A, Boneya D, Campos-Nonato I, Castaneda-Orjuela C, Catala-Lopez F, Chiang P, Chibueze C, Chitheer A, Choi JY, Cowie B, Damtew S, das Neves J, Dey S, Dharmaratne S, Dhillon P, Ding E, Driscoll T, Ekwueme D, Endries AY, Farvid M, Farzadfar F, Fernandes J, Fischer F, TT GH, Gebru A, Gopalani S, Hailu A, Horino M, Horita N, Husseini A, Huybrechts I, Inoue M, Islami F, Jakovljevic M, James S, Javanbakht M, Jee SH, Kasaeian A, Kedir MS, Khader YS, Khang YH, Kim D, Leigh J, Linn S, Lunevicius R, El Razek 
HMA, Malekzadeh R, Malta DC, Marcenes W, Markos D, Melaku YA, Meles KG, Mendoza W, Mengiste DT, Meretoja TJ, Miller TR, Mohammad KA, Mohammadi A, Mohammed S, Moradi-Lakeh M, Nagel G, Nand D, Le Nguyen Q, Nolte S, Ogbo FA, Oladimeji KE, Oren E, Pa M, Park EK, Pereira DM, Plass D, Qorbani M, Radfar A, Rafay A, Rahman M, Rana SM, Soreide K, Satpathy M, Sawhney M, Sepanlou SG, Shaikh MA, She J, Shiue I, Shore HR, Shrime MG, So S, Soneji S, Stathopoulou V, Stroumpoulis K, Sufiyan MB, Sykes BL, Tabares-Seisdedos R, Tadese F, Tedla BA, Tessema GA, Thakur JS, Tran BX, Ukwaja KN, Uzochukwu BSC, Vlassov VV, Weiderpass E, Wubshet Terefe M, Yebyo HG, Yimam HH, Yonemoto N, Younis MZ, Yu C, Zaidi Z, Zaki MES, Zenebe ZM, Murray CJL and Naghavi M: Global, regional, and national cancer incidence, mortality, years of life lost, years lived with disability, and disability-adjusted life-years for 32 cancer groups, 1990 to 2015: A systematic analysis for the global burden of disease study. JAMA Oncol 3(4): 524-548, 2017. PMID: 27918777. DOI: $10.1001 /$ jamaoncol.2016.5688

2 Bang YJ, Van Cutsem E, Feyereislova A, Chung HC, Shen L, Sawaki A, Lordick F, Ohtsu A, Omuro Y, Satoh T, Aprile G, Kulikov E, Hill J, Lehle M, Ruschoff J, Kang YK and To GATI: Trastuzumab in combination with chemotherapy versus chemotherapy alone for treatment of her2-positive advanced gastric or gastro-oesophageal junction cancer (toga): A phase 3, open-label, randomised controlled trial. Lancet 376(9742): 687697, 2010. PMID: 20728210. DOI: 10.1016/S0140-6736(10) 61121-X

3 Wilke H, Muro K, Van Cutsem E, Oh SC, Bodoky G, Shimada Y, Hironaka S, Sugimoto N, Lipatov O, Kim TY, Cunningham D, Rougier P, Komatsu Y, Ajani J, Emig M, Carlesi R, Ferry D, Chandrawansa K, Schwartz JD, Ohtsu A and Group RS: Ramucirumab plus paclitaxel versus placebo plus paclitaxel in patients with previously treated advanced gastric or gastrooesophageal junction adenocarcinoma (rainbow): A double-blind, randomised phase 3 trial. Lancet Oncol 15(11): 1224-1235, 2014. PMID: 25240821. DOI: 10.1016/S1470-2045(14)70420-6

4 Kang YK, Boku N, Satoh T, Ryu MH, Chao Y, Kato K, Chung HC, Chen JS, Muro K, Kang WK, Yeh KH, Yoshikawa T, Oh SC, Bai LY, Tamura T, Lee KW, Hamamoto Y, Kim JG, Chin K, Oh DY, Minashi K, Cho JY, Tsuda M and Chen LT: Nivolumab in patients with advanced gastric or gastro-oesophageal junction cancer refractory to, or intolerant of, at least two previous chemotherapy regimens (ono-4538-12, attraction-2): A randomised, double-blind, placebo-controlled, phase 3 trial. Lancet 390(10111): 2461-2471, 2017. PMID: 28993052. DOI: 10.1016/S0140-6736(17)31827-5

5 Kang BW, Baek DW, Kang H, Baek JH and Kim JG: Novel therapeutic approaches for epstein-barr virus associated gastric cancer. Anticancer Res 39(8): 4003-4010, 2019. PMID: 31366481. DOI: 10.21873 /anticanres.13555

6 Morin PJ: Claudin proteins in human cancer: Promising new targets for diagnosis and therapy. Cancer Res 65(21): 9603-9606, 2005. PMID: 16266975. DOI: 10.1158/0008-5472.CAN-05-2782

7 Swisshelm K, Macek R and Kubbies M: Role of claudins in tumorigenesis. Adv Drug Deliv Rev 57(6): 919-928, 2005. PMID: 15820559. DOI: 10.1016/j.addr.2005.01.006

8 Angelow S, Ahlstrom R and Yu AS: Biology of claudins. Am J Physiol Renal Physiol 295(4): F867-876, 2008. PMID: 18480174. DOI: 10.1152 /ajprenal.90264.2008
9 Escudero-Esparza A, Jiang WG and Martin TA: The claudin family and its role in cancer and metastasis. Front Biosci (Landmark Ed) 16: 1069-1083, 2011. PMID: 21196219. DOI: $10.2741 / 3736$

10 Sahin U, Koslowski M, Dhaene K, Usener D, Brandenburg G, Seitz G, Huber C and Tureci O: Claudin-18 splice variant 2 is a pan-cancer target suitable for therapeutic antibody development. Clin Cancer Res 14(23): 7624-7634, 2008. PMID: 19047087. DOI: 10.1158/1078-0432.CCR-08-1547

11 Al-Batran SE, Schuler MH, Zvirbule Z, Manikhas G, Lordick F, Rusyn A, Vynnyk Y, Vynnychenko I, Fadeeva N, Nechaeva M, Dudov A, Gotovkin E, Pecheniy A, Bazin I, Bondarenko I, Melichar B, Mueller C, Huber C, Tureci O and Sahin U: Fast: An international, multicenter, randomized, phase II trial of epirubicin, oxaliplatin, and capecitabine (eox) with or without imab362, a first-in-class anti-cldn18.2 antibody, as first-line therapy in patients with advanced cldn18.2+ gastric and gastroesophageal junction (gej) adenocarcinoma. J Clin Oncol 34: LBA4001, 2016. DOI: 10.1200/JCO.20165.34.18_suppl.LBA4001

12 Rohde C, Yamaguchi R, Mukhina S, Sahin U, Itoh K and Tureci $\mathrm{O}$ : Comparison of claudin 18.2 expression in primary tumors and lymph node metastases in japanese patients with gastric adenocarcinoma. Jpn J Clin Oncol, 2019. PMID: 31087075. DOI: $10.1093 /$ jjco/hyz068

13 Dottermusch M, Kruger S, Behrens HM, Halske C and Rocken C: Expression of the potential therapeutic target claudin-18.2 is frequently decreased in gastric cancer: Results from a large caucasian cohort study. Virchows Arch, 2019. PMID: 31332522. DOI: $10.1007 / \mathrm{s} 00428-019-02624-7$

14 Edge SB and Compton CC: The american joint committee on cancer: The 7th edition of the ajcc cancer staging manual and the future of tnm. Ann Surg Oncol 17(6): 1471-1474, 2010. PMID: 20180029. DOI: 10.1245/s10434-010-0985-4

15 Sakuramoto S, Sasako M, Yamaguchi T, Kinoshita T, Fujii M, Nashimoto A, Furukawa H, Nakajima T, Ohashi Y, Imamura H, Higashino M, Yamamura Y, Kurita A, Arai K and Group A-G: Adjuvant chemotherapy for gastric cancer with s-1, an oral fluoropyrimidine. N Engl J Med 357(18): 1810-1820, 2007. PMID: 17978289. DOI: 10.1056/NEJMoa072252

16 Bang YJ, Kim YW, Yang HK, Chung HC, Park YK, Lee KH, Lee KW, Kim YH, Noh SI, Cho JY, Mok YJ, Kim YH, Ji J, Yeh TS, Button P, Sirzen F, Noh SH and investigators Ct: Adjuvant capecitabine and oxaliplatin for gastric cancer after $\mathrm{d} 2$ gastrectomy (classic): A phase 3 open-label, randomised controlled trial. Lancet 379(9813): 315-321, 2012. PMID: 22226517. DOI: 10.1016/S0140-6736(11)61873-4

17 Yang H, Hong D, Cho SY, Park YS, Ko WR, Kim JH, Hur H, Lee J, Kim SJ, Kwon SY, Lee JH, Park DY, Song KS, Chang H, Ryu MH, Cho KS, Kang JW, Kook MC, Thiessen N, He A, Mungall A, Han SU and Kim HK: Rhogap domain-containing fusions and ppapdc1a fusions are recurrent and prognostic in diffuse gastric cancer. Nat Commun 9(1): 4439, 2018. PMID: 30361512. DOI: 10.1038/s41467-018-06747-4

18 Yao F, Kausalya JP, Sia YY, Teo AS, Lee WH, Ong AG, Zhang Z, Tan JH, Li G, Bertrand D, Liu X, Poh HM, Guan P, Zhu F, Pathiraja TN, Ariyaratne PN, Rao J, Woo XY, Cai S, Mulawadi FH, Poh WT, Veeravalli L, Chan CS, Lim SS, Leong ST, Neo SC, Choi PS, Chew EG, Nagarajan N, Jacques PE, So JB, Ruan X, Yeoh KG, Tan P, Sung WK, Hunziker W, Ruan Y and Hillmer AM: Recurrent fusion genes in gastric 
cancer: Cldn18-arhgap26 induces loss of epithelial integrity. Cell Rep 12(2): 272-285, 2015. PMID: 26146084. DOI: 10.1016/j.celrep.2015.06.020

19 Tanaka A, Ishikawa S, Ushiku T, Yamazawa S, Katoh H, Hayashi A, Kunita A and Fukayama M: Frequent cldn18-arhgap fusion in highly metastatic diffuse-type gastric cancer with relatively early onset. Oncotarget 9(50): 29336-29350, 2018. PMID: 30034621. DOI: 10.18632/oncotarget.25464

20 Steven A, Leisz S, Massa C, Iezzi M, Lattanzio R, Lamolinara A, Bukur J, Muller A, Hiebl B, Holzhausen H and Seliger B: Her-2/neu mediates oncogenic transformation via altered creb expression and function. Mol Cancer Res 11(11): 1464-1477, 2016. PMID: 24025972. DOI: 10.1158/1541-7786.MCR-130125

21 Schuler MH, Al-Batran SE, Zvirbule Z, Manikhas G, Lordick F, Rusyn A, Vynnychenko I, Dudov A, Bazin I, Melichar B, Dhaene $\mathrm{K}$, Wiechen K, Maurus D, Tureci O and Sahin U: Expression of claudin 18.2 and her2 in gastric, gastroesophageal junction, and esophageal cancers: Results from the fast study. J Clin Oncol 35: 4038-4038, 2017. DOI: 10.1200/JCO.2017.35.15_suppl.4038
22 Moran D, Maurus D, Rohde C and Arozullah A: Prevalence of cldn18.2, her2, and pd-11 in gastric cancer samples. Ann Oncol 29: viii14-viii57, 2018. DOI: 10.1093/annonc/mdy269

23 Jun KH, Kim JH, Jung JH, Choi HJ and Chin HM: Expression of claudin-7 and loss of claudin-18 correlate with poor prognosis in gastric cancer. Int J Surg 12(2): 156-162, 2014. PMID: 24333468. DOI: 10.1016/j.ijsu.2013.11.022

24 Oshima T, Shan J, Okugawa T, Chen X, Hori K, Tomita T, Fukui $\mathrm{H}$, Watari $\mathrm{J}$ and Miwa H: Down-regulation of claudin-18 is associated with the proliferative and invasive potential of gastric cancer at the invasive front. PLoS One 8(9): e74757, 2013. PMID: 24073219. DOI: 10.1371/journal.pone.0074757

Received October 15, 2019

Revised October 26, 2019

Accepted October 29, 2019 